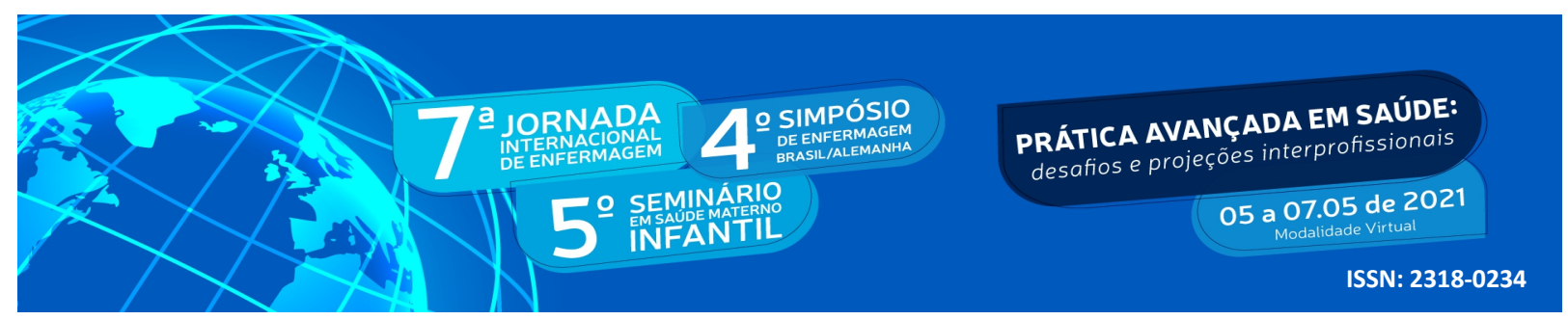

DOI: http://doi.org/10.48195/jie2021-019

\title{
PROMOÇÃO DO CUIDADO EM SAÚDE DAS PESSOAS IDOSAS NO ATENDIMENTO GERIÁTRICO E GERONTOLÓGICO ${ }^{1}$
}

\author{
Maria Eduarda Paniz ${ }^{2}$; Angélica Farinha ${ }^{3}$; Naiana Oliveira dos Santos ${ }^{4}$; Maria Helena \\ Gehlen $^{5}$
}

\section{RESUMO}

Objetiva-se descrever a promoção do cuidado em saúde às pessoas idosas no atendimento geriátrico e gerontológico. Método: pesquisa tipo qualitativa, em nível exploratório-descritivo, com abordagem do materialismo histórico e dialético. Os dados foram obtidos por meio de observação sistemática, entrevista semiestruturada e analisados conforme a Técnica de Análise do Conteúdo. O projeto foi aprovado pelo Comitê de Ética em Pesquisa com número de registro 2.202.003. Desta forma, foi possível refletir o acerca da necessidade da qualificação geriátrica gerontológica dos profissionais na prevenção de agravos associados ao processo de envelhecimento e promoção da saúde da pessoa idosa. Palavras-chave: Enfermagem Geriátrica; Idoso; Profissionais da Saúde.

\begin{abstract}
The objective is to describe the promotion of health care for the elderly in geriatric and gerontological care.Method: qualitative research, on an exploratory-descriptive level, with an approach to historical and dialectical materialism.The data were obtained through systematic observation, semi-structured interviews and analyzed according to the Content Analysis Technique. The project was approved by the Research Ethics Committee with registration number 2,202,003.Thus, it was possible to reflect on the need for geriatric gerontological qualification of professionals in preventing diseases associated with the aging process and promoting the health of the elderly.
\end{abstract}

Key Words: Geriatric Nursing; Elderly; Health Professionals.

\footnotetext{
1 Artigo de pesquisa exploratória e descritiva na abordagem qualitativa.

2 Estudante do Curso de Enfermagem. Universidade Franciscana - UFN. E-mail: mariafpaniz@gmail.com

${ }^{3}$ Estudante do Curso de Enfermagem. Universidade Franciscana - UFN. E-mail: angelicaluciom2011@hotmail.com

${ }^{4}$ Professora Dra. Do Curso de Enfermagem. Universidade Franciscana - UFN. E-mail: naiana.santos@ufn.edu.br 35 Orientadora Professora Dra. Do Curso de Enfermagem Universidade Franciscana - UFN. E-mail: gehlenmh@gmail.com
} 


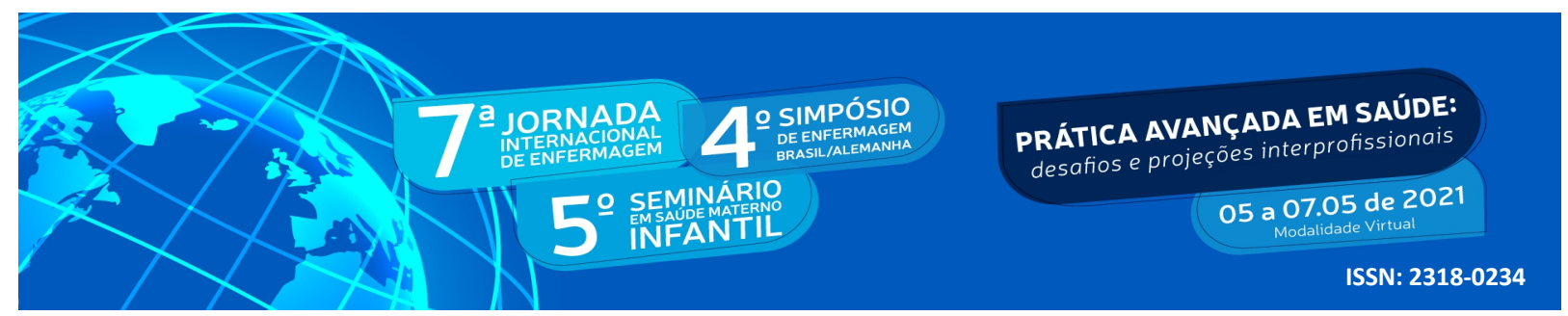

\section{INTRODUÇ̃̃̃O}

O envelhecimento populacional acontece de forma acelerada, tanto em países desenvolvidos, quanto em países em desenvolvimento. Assim, estima-se um incremento anual médio de mais de 1 milhão de pessoas idosas, resultado da diminuição das taxas de fecundidade e de mortalidade, do aumento da expectativa de vida, da melhoria dos serviços de saúde e do uso de novas tecnologias. Segundo Miranda (2016), o índice de envelhecimento aumentará de 10,6 em 1920 para 152,6 em 2040, à vista disso, os idosos de hoje, têm o costume de realizar atividades físicas, alimentação saudável, e principalmente, entendem que envelhecer não é adoecer e deixar de viver a vida.

A idade avançada traz consigo algumas comorbidades habituais que podem ser tratadas e acompanhadas, no atendimento geriátrico gerontológico. Consequentemente, o processo de envelhecimento gera adequações na promoção do cuidado à saúde da pessoa, pois muitas vezes, motiva o uso frequente dos serviços de saúde devido ao surgimento de algumas doenças de origem orgânicas ou comportamentais que poderão comprometer sua funcionalidade. Deste modo, relaciona-se a importância de os profissionais da saúde estarem qualificados para a promoção do cuidado em saúde desses idosos, no atendimento geriátrico gerontológico na promoção de saúde, conforto e bem estar para profilaxia de maiores comorbidades (DIAS et al, 2014).

Entretanto, apesar dos avanços das políticas públicas de saúde, na promoção integral da saúde da pessoa idosa há necessidade de melhorias no cuidado especializado no atendimento geriátrico e gerontológico para um envelhecimento ativo e saudável. Segundo Martins (2007), a Política Nacional do Idoso, a Política Nacional da Saúde da Pessoa Idosa e o Estatuto do Idoso e o Pacto da Vida, são instrumentos que garantem a proteção a esse grupo populacional, agregando-os na condição de cidadãos que, como os demais, merecem uma atenção digna e saudável, considerados parceiros ativos no desenvolvimento dessa política. $\mathrm{O}$ qual significa que o perfil biopsicossocial do ser humano passa a exigir novos enfoques culturais, sociais e de saúde.

\section{OBJETIVO}




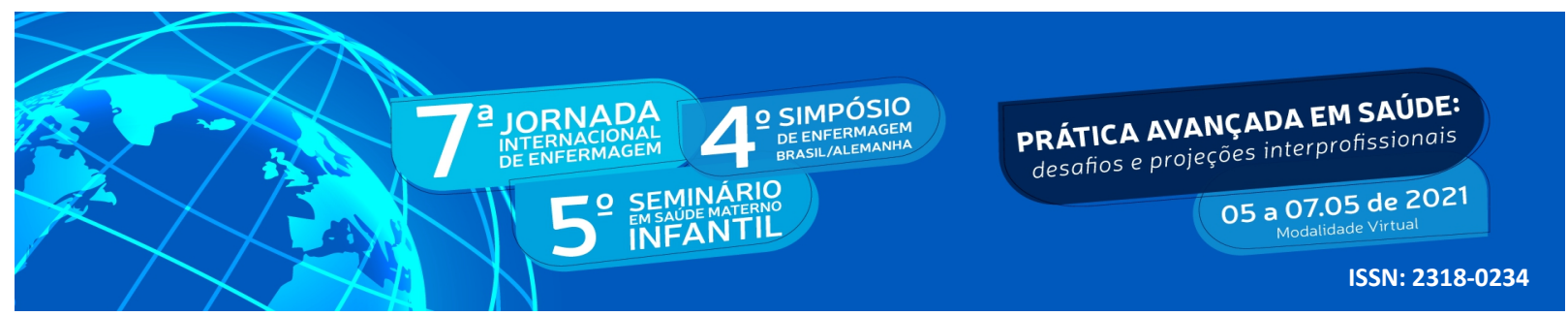

Esta pesquisa tem como objetivo descrever como ocorre a promoção do cuidado em saúde das pessoas idosas em uma Instituição de Longa Permanência para Idosos, desenvolvido por profissionais da área da saúde no atendimento geriátrico e gerontológico.

\section{METODOLOGIA}

Trata-se de um estudo do tipo qualitativo, em nível exploratório-descritivo, com abordagem do materialismo histórico e dialético. Os dados foram obtidos no período do segundo semestre de 2019, durante os meses de agosto a dezembro, por meio de observação sistemática, entrevista semiestruturada e analisados conforme a Técnica de Análise do Conteúdo, de Bardin (2016) . De tal modo, por meio da pré-análise foram determinadas as unidades de registro: palavras-chave ou frases, unidades de contexto, recortes, forma de categorização e conceitos teóricos gerais que orientaram a análise. Portanto, levou-se em conta a questão central e o objetivo da pesquisa na realização da codificação.

Após foi realizado a exploração do material, que consistiu na transformação dos dados iniciais obtidos, objetivando a compreensão do texto a partir do seu núcleo de sentido codificado, em que procedeu-se com o recorte do texto em unidades de registro sendo realizada a classificação e agregação dos dados.

Para a análise, interpretação e tratamento dos resultados obtidos: houve a categorização dos dados por meio da interpretação, correlacionando os achados investigados à luz dos referenciais teórico e metodológico que fundamentaram a pesquisa.

A pesquisa foi feita na Instituição de Longa Permanência para idosas Lar das Vovozinhas. Os participantes da pesquisa eram acadêmicos da área de saúde, constituintes e voluntários de um projeto de promoção do cuidado para as idosas, que possui o nome de Viva +. A pesquisa foi aprovada pelo Comitê de Ética em Pesquisa com número de registro 2.202.003, seguindo os pressupostos da resolução Conselho Nacional de Saúde n 466 ,de 12 de dezembro de 2012. 


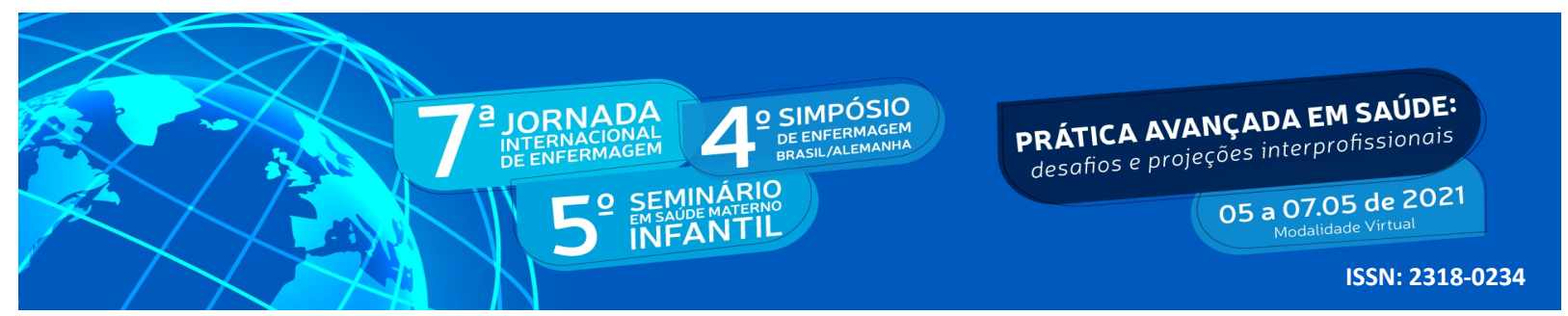

\section{RESULTADOS E DISCUSSÃO}

Identificou-se que os profissionais de saúde desejam buscar qualificar suas práticas e saberes com especialização para melhoria da promoção do cuidado com ênfase ao auxílio e manutenção das atividades básicas de vida diária, na funcionalidade e no bem-estar da pessoa idosa. Verificou-se que há desejo dos profissionais de saúde em desenvolverem discussões e estratégias interdisciplinares, em respeito à autonomia, práticas, saberes no atendimento geriátrico gerontológico. Observou-se que a relação de apoio emocional e empenho dos profissionais, colabora com a cidadania e direitos das pessoas idosas e contribui na qualidade do atendimento geriátrico gerontológico. Mas quando envolvida pela impaciência oriunda do despreparo profissional há a colaboração para uma relação iatrogênica que compromete o respeito e a autonomia. Segundo Silva (2019), os objetivos principais do serviço da enfermagem, são: assistir ao cliente, em suas necessidades humanas e básicas; promover ensino, pesquisa e aprimoramento profissional, conforme o código de ética; organizar a assistência de enfermagem, regida pelo gerente da enfermagem. Além disso, os profissionais de saúde estão acostumados ao olhar centrado nos agravos apresentados pelas idosas, porém, é necessário ampliar a visão profissional, para reconhecer os contextos e condições de saúde (PIEXAK et al, 2012).

Desta maneira, a qualificação profissional e a comunicação terapêutica, acolhedora, alegre, descontraída, influência para mudanças de comportamento no acompanhamento geriátrico gerontológico, possibilitando a pessoa idosa sentir-se como um ser humano digno e capaz de solucionar seus problemas (MACHADO; BRÊTAS, 2016). Pereira (2017), relata que a humanização no trabalho de enfermagem é uma necessidade atual, que assim, exige que o profissional repense sua ação. A humanização não é apenas de outro tipo de cuidado, mas também englobam situações de respeito, apoio, diálogo e empatia, onde estes precisam da efetividade na atuação do enfermeiro.

Evidenciou-se que na promoção do cuidado em saúde das pessoas idosas desenvolvido por profissionais no atendimento geriátrico e gerontológico é importante a qualificação profissional que permite criar estratégias, tanto na relação com um usuário, como nas práticas de saúde, na busca de interferência no estilo de vida no contexto, das condições 


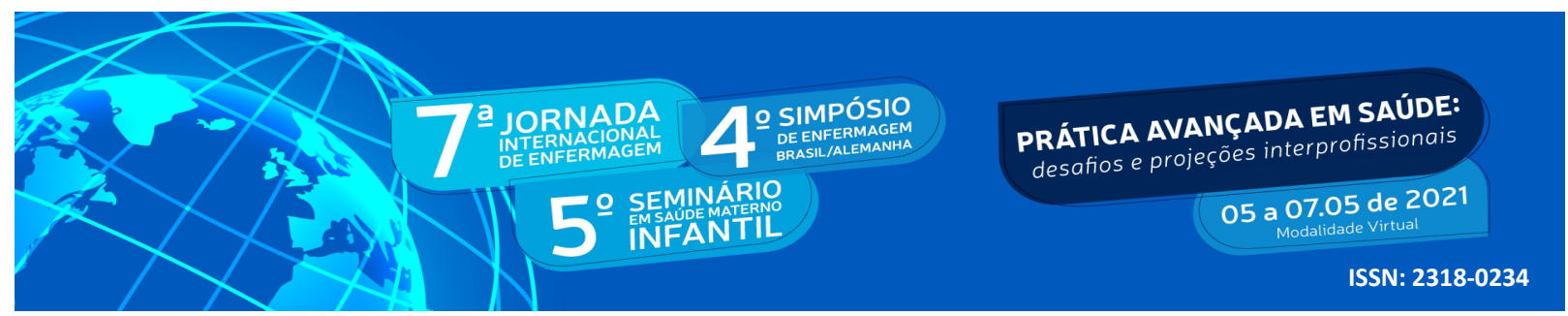

econômicas, sociais e ambientais. Segundo Lima (2019), é inquestionável a importância dos cuidados específicos dos serviços de enfermagem ao assistir ao idoso, pois envolvem conhecimentos técnicos, psicológicos, culturais, comunicacionais, informacionais, além de considerar a plena condição da essência humana.

Ressalta-se que a falta de investimentos públicos e recursos humanos, tanto na quantidade, quanto na qualidade para o atendimento às necessidades específicas da população da pessoa idosa. Portanto, a qualidade nas relações interpessoais com o usuário por meio da qualificação profissional gera transmissão de confiança, troca de emoções positivas e possibilita o melhor atendimento e cuidado ao profissional da saúde e ao idoso. (ARAÚJO; BARBOSA, 2010).

\section{CONCLUSÃO}

A promoção do cuidado em saúde da pessoa idosa desenvolvido pelos profissionais de saúde no atendimento geriátrico gerontológico é um desafio no cotidiano frente a qualificação profissional, avaliação, acompanhamento e intervenções ou prescrições interdisciplinares. Destacando o cuidado como a atenção, a escuta qualificada, e o atendimento das necessidades geriátrica e gerontológica. Entretanto, pode haver algumas dificuldades no relacionamento, como a impaciência profissional, educação em saúde de forma autoritária, que promove a iatrogenia e prejudica a ambiência interprofissional.

\section{REFERÊNCIAS BIBLIOGRÁFICAS}

ARAÚJO, M.A.S. et al. Relação entre o profissional de saúde da família e o idoso. Esc. Anna Nery, vol.14 no.4 Rio de Janeiro out./dez. 2010.

BARDIN, L. Análise de Conteúdo. 3. ed. Lisboa, 2016.

BRASIL. Diretrizes e normas regulamentadoras de pesquisa envolvendo seres humanos. Resolução no 466, Brasília, 12 de dezembro de 2012. 


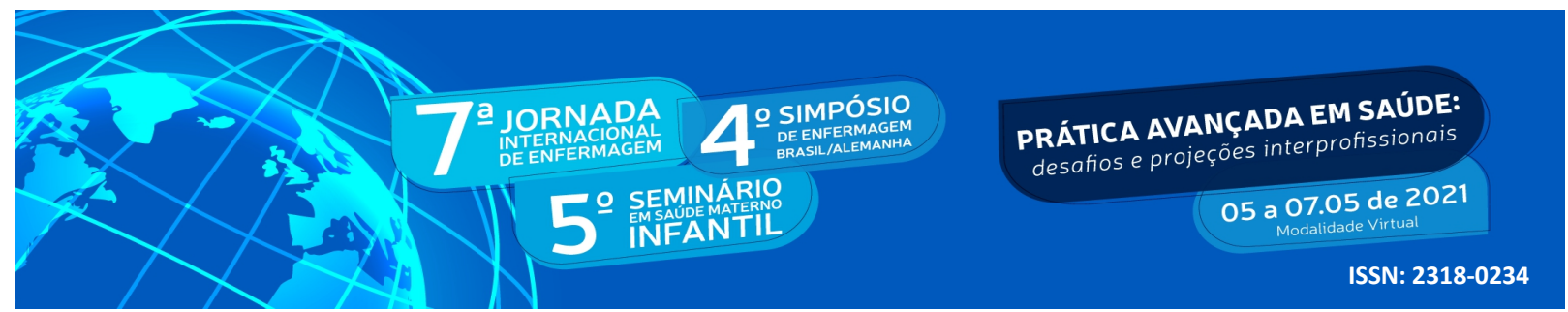

DIAS, K. C. O. et al. O cuidado em enfermagem direcionado para a pessoa idosa: revisão integrativa. Rev. Enferm. UFPE on line. ,v. 8, n. 5, p. 1337-1346, Recife, 2014.

LIMA, T. et. al. Humanização na atenção básica de saúde na percepção de idosos. Saúde Soc. v.23, n.1, p. 265-276, São Paulo, 2014.

MACHADO, A. C. A.; BRÊTAS, A. C. P. Comunicação não-verbal de idosos frente ao processo de dor. Rev. bras. enferm., v. 59 n. 2, Mar./Apr. Brasília, 2016.

MARTINS, J.J. et al. Políticas públicas de atenção à saúde do idoso: reflexão acerca da capacitação dos profissionais de saúde para o cuidado com o idoso. Rev. bras. geriatr. gerontol. vol.10 no.. Sept./Dec. Rio de Janeiro, 2007.

MIRANDA, G. M. D.; MENDES. A. C. G.; SILVA, A. L. A. O envelhecimento populacional brasileiro: desafios e consequências sociais atuais e futuras. Rev. Bras. Geriatr. Gerontol., v. 19, n. 3, p. 507-519, Rio de Janeiro, 2016.

PEREIRA, M. O. Prática Assistencial de Enfermagem: humanização no cuidar. Temas de Saúde. V.17(3): 163-173. João Pessoa, 2017.

PIEXAK D. G., et al. Percepção de profissionais de saúde em relação ao cuidado a pessoas idosas institucionalizadas. Rev. bras. geriatr. gerontol. v.15 no.2 Rio de Janeiro, 2012.

SILVA, K. M.; SANTOS, S. M. A. A consulta de enfermagem ao idoso na estratégia de saúde da família: desafios e possibilidades. Cienc Cuid Saude, v. 13, n. 1, p. 49-57, Jan/Mar. São Paulo, 2014. 